\title{
An intracerebral exploration of functional connectivity during word production
}

\author{
Amandine Grappe ${ }^{1,2}$. Sridevi V. Sarma ${ }^{1}$ - Pierre Sacré ${ }^{1}$. Jorge González-Martínez ${ }^{3}$. Catherine Liégeois-Chauvel ${ }^{3,4}$. \\ F.-Xavier Alario 5
}

Received: 15 January 2018 / Revised: 25 September 2018 / Accepted: 28 September 2018

(c) Springer Science+Business Media, LLC, part of Springer Nature 2018

\begin{abstract}
Language is mediated by pathways connecting distant brain regions that have diverse functional roles. For word production, the network includes a ventral pathway, connecting temporal and inferior frontal regions, and a dorsal pathway, connecting parietal and frontal regions. Despite the importance of word production for scientific and clinical purposes, the functional connectivity underlying this task has received relatively limited attention, and mostly from techniques limited in either spatial or temporal resolution. Here, we exploited data obtained from depth intra-cerebral electrodes stereotactically implanted in eight epileptic patients. The signal was recorded directly from various structures of the neocortex with high spatial and temporal resolution. The neurophysiological activity elicited by a picture naming task was analyzed in the time-frequency domain $(10-150 \mathrm{~Hz})$, and functional connectivity between brain areas among ten regions of interest was examined. Task related-activities detected within a network of the regions of interest were consistent with findings in the literature, showing task-evoked desynchronization in the beta band and synchronization in the gamma band. Surprisingly, long-range functional connectivity was not particularly stronger in the beta than in the high-gamma band. The latter revealed meaningful subnetworks involving, notably, the temporal pole and the inferior frontal gyrus (ventral pathway), and parietal regions and inferior frontal gyrus (dorsal pathway). These findings are consistent with the hypothesized network, but were not detected in every patient. Further research will have to explore their robustness with larger samples.
\end{abstract}

Keywords Speech $\cdot$ Language $\cdot$ Dorsal stream $\cdot$ Ventral stream $\cdot$ Stereo-electroencephalography $\cdot$ Functional connectivity

\section{Introduction}

Contemporary views about language processing have evolved from the classic ideas developed by Broca (1861),

Action Editor: Byron Yu

F.-Xavier Alario

francois-xavier.alario@univ-amu.fr

1 Institute for Computational Medicine, Johns Hopkins University, Baltimore, MD, USA

2 Interfaculty Institute of Bioengineering, Ecole Polytechnique Fédérale de Lausanne, Lausanne, Switzerland

3 Cleveland Clinic Neurological Institute, Epilepsy Center, Cleveland, OH, USA

4 INS, Institute of System Neuroscience, Aix Marseille University, INSERM, Marseille, France

5 LPC, Aix Marseille University, CNRS, Marseille, France
Wernicke (1969), and Lichtheim (1885), or Geschwind (1970), to models and descriptions that are substantially more specific and complex (Tremblay and Dick 2016). Classic models highlighted a distinction between language comprehension and production, with corresponding specializations in the posterior temporal cortex and the inferior frontal gyrus of the language-dominant hemisphere. Today, language processing is understood as a distributed global network of neural structures that are devoted to diverse subprocesses (Price 2012), functionally organized in multiple processing streams (Poeppel et al. 2012) through dorsal and ventral anatomical pathways (Fig. 1; e.g. Petrides 2014).

\subsection{Anatomo-functional divisions}

Consider a word production task in which pictured objects are named out-loud (Snodgrass and Yuditsky 1996), and many regions of the language network are recruited (e.g. Hickok 2012; Roelofs 2014; Ueno et al. 2011). Early visual processing stages recruit occipital and temporal 


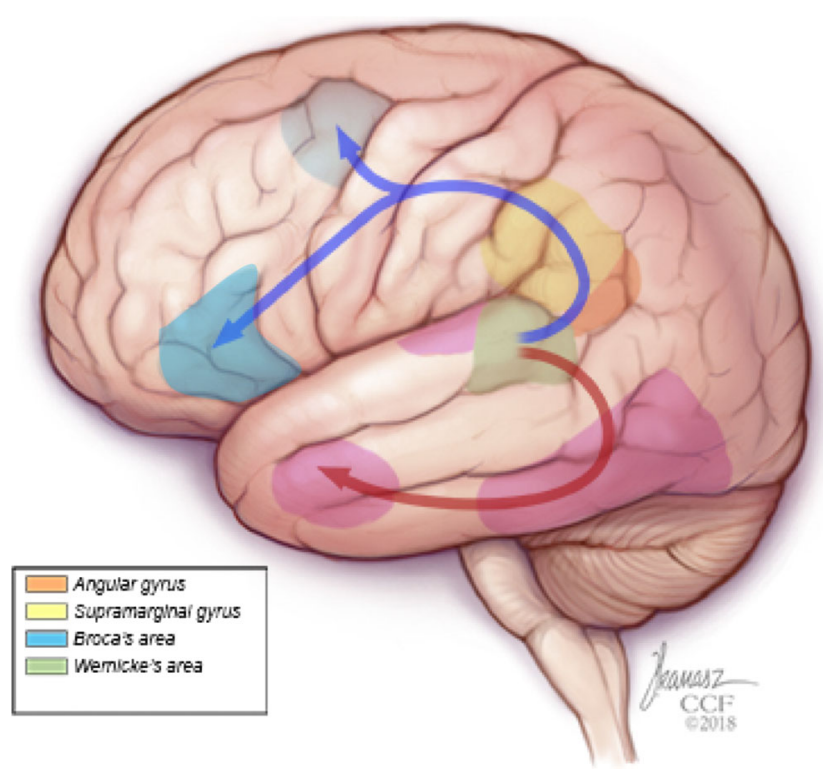

Fig. 1 Schematic representation of dorsal (blue) and ventral (pink) streams involved in language processing

regions (e.g., fusiform gyrus and the medial temporal gyrus; Goodale and Milner 1992; Grill-Spector and Weiner 2014). Semantic processing recruits the temporal pole and the angular gyrus (Binder and Desai 2011; Lambon-Ralph et al. 2016). Word processing is often associated with middle temporal lobe (reviewed in Indefrey 2011), with a possible role of medial temporal lobe regions (e.g., hippocampus) in visual word retrieval (Hamamé et al. 2014; Llorens et al. 2016; see also Covington and Duff 2016; Moscovitch et al. 2016; Piai et al. 2016). Phonological and articulatory processes are associated to parietal and prefrontal regions (e.g., Dell et al. 2013; Fridriksson et al. 2016; Rogalsky et al. 2015; Schwartz et al. 2012; Han et al. 2016).

\subsection{Network pathways}

Such regions are connected through various anatomical pathways (details in Petrides 2014). The visual ventral pathway connects occipital regions to inferior temporal regions, onwards to the temporal pole and mesial areas (Clarke and Tyler 2015). Temporal and frontal areas are connected dorsally, through parietal areas, by the middle longitudinal fasciculus (Catani et al. 2005), and by the arcuate fasciculus, thought to play a crucial role, since the classic Geshwind model, in connecting superior temporal regions with prefrontal areas (Catani and Mesulam 2008). The areas in the parietal lobe (e.g. Angular Gyrus and Supra-Marginal Gyrus) are differentially connected to subregions within the prefrontal area (Catani and Mesulam 2008; Petrides 2014; Parlatini et al. 2017) by the major branches of the superior longitudinal fasciculus, which is not always distinguished from the arcuate fasciculus cited earlier. Finally, anterior temporal areas are also anatomically connected to the different sub-regions in inferior frontal gyrus by an alternative ventral pathway that includes the uncinate fasciculus and the extreme capsule fasciculus (Makris and Pandya 2009; Papagno 2011).

\subsection{Task-related functional connectivity}

The evidence for the pathways described above comes from comparative studies in macaque monkeys (e.g., Petrides and Pandya 2009), and, in humans, from metrics derived from structural diffusion tensor imaging (DTI) connectivity and from functional magnetic resonance imaging (fMRI) resting-state connectivity (e.g., Margulies and Petrides 2013; Fuertinger et al. 2015; Saur et al. 2008; Sheldon et al. 2016; Sheldon and Moscovitch 2012; Uddin et al. 2010). Comparatively, there are relatively few studies in which functional connectivity analysis are directly derived from word production tasks. These studies are highly diverse (Table 1), leaving many open questions regarding functional connectivity during word production. Of particular interest to our own approach are previous explorations based on intracerebral recordings, which provide neurophysiological signals with high spatial and temporal resolutions (see also Llorens et al. 2011). These have shown that the network dynamic structure is substantially modulated by broad task requirements (e.g., visual vs. auditory input, or spoken vs. signed output: Korzeniewska et al. 2011; Collard et al. 2016), while still reflecting the expected language pathways described above (Flinker et al. 2015), and sometimes refining them (Rolston and Chang in press).

\subsection{The current study}

The hypothesis tested here is that the structural pathways reviewed above are dynamically operational during cognitive language tasks. If such is the case, the dynamic functional activity elicited during a word-production task should reveal statistical dependencies between brain regions. Based on our past research, we were particularly interested in identifying regions that were simultaneously and similarly active during the task (Dubarry et al. 2017). Given this goal, our exploration purposely avoided exploring metrics that involve delays between regions. Instead of focusing on a particular frequency band, as was done there, here we explored separately the similarity of functional activity between pairs of brain structures in the $\beta$ band $(12-30 \mathrm{~Hz})$ and the $\gamma_{\text {high }}$ band $(70-150 \mathrm{~Hz})$ on a trialby-trial basis. Electrophysiological oscillations in different frequency bands co-occur with perceptual, cognitive and sensory-motor tasks, and it is acknowledged that they can correlate with different cognitive states (Wang 2010). The 


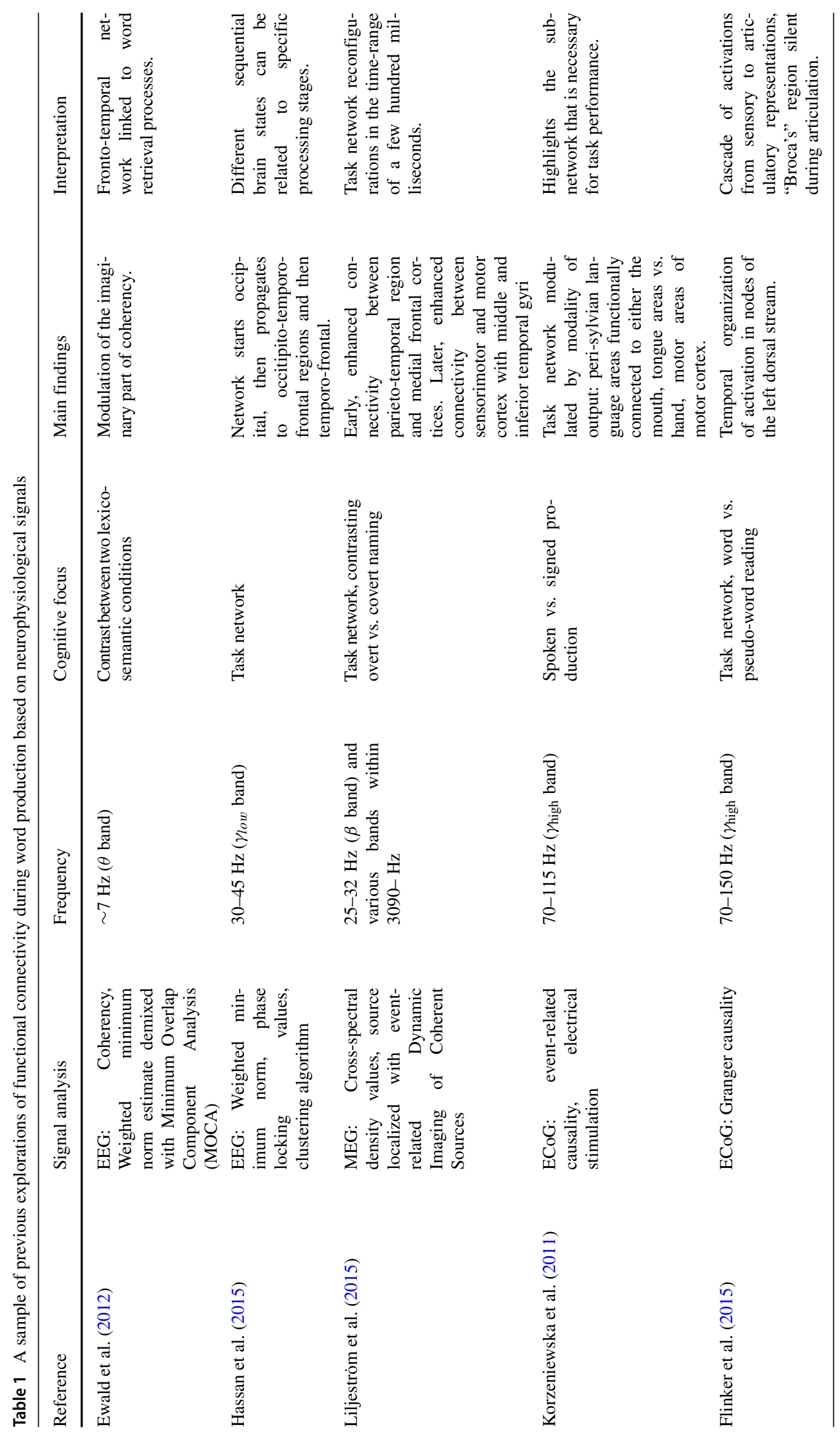


specific roles of these oscillations in language remains largely an open question. The $\gamma_{\text {high }}$ band was a focus of this study because it has been associated with language processing, particularly in intracerebral recordings (discussed in Lachaux et al. 2012; see also Gaona et al. 2011). The $\beta$ band was another focus of this study because it has been previously associated with various linguistics processes, including retrieval and sensorimotor processes (e.g., Liljeström et al. 2015; Piai et al. 2015). Low-frequency oscillatory activity has been claimed to be the key mechanism for long-range neuronal interaction and information transfer (Donner and Siegel 2011). $\beta$ and $\gamma_{\text {high }}$ synchronization might be differentially involved in top-down vs. bottom-up processes within certain cognitive processes (Fries 2015).

In short, we sought to quantify similar co-activations among brain regions during word production across frequency bands.

\section{Materials and methods}

The experiment was performed at the Epilepsy Center of Cleveland Clinic, Ohio, United States of America. It had been approved by the local Institutional Review Board with approval number 16-466. All participants gave their informed written consent before being enrolled.

\subsection{Participants}

The participants were eight epileptic patients (3 men, 5 women; $42.5 \pm 11.5$ years old) that did not respond to standard drug treatments and that had been implanted with intracerebral electrodes for pre-surgical diagnostic purposes. The choice of electrode location had been based on a pre-implantation patient management conference and was made independently of the present study (Fig. 2). Criteria for patients undergoing SEEG implantation were reviewed by clinicians to determine patient eligibility for enrollment in the current study. If the patient met study criteria, research staff not involved in the surgery implantation or post-surgical care contacted the patient for potential participation in the study. Participation in the study did not alter the patients' clinical care, particularly it had strictly no influence on the originally planned SEEG procedure (Johnson et al. 2014). The participants' demographics are presented on Table 2.

\subsection{Behavioral task}

The cognitive experiment was based on a previous study performed by (Llorens et al. 2014). Word production was elicited in a picture naming task involving 36 pictures of common objects (Fig. 3). Following a common 
Fig. 2 Robotic SEEG technique. Planning SEEG trajectories using the robotic software and pre-operative volumetric contrasted T1 sequence MRI

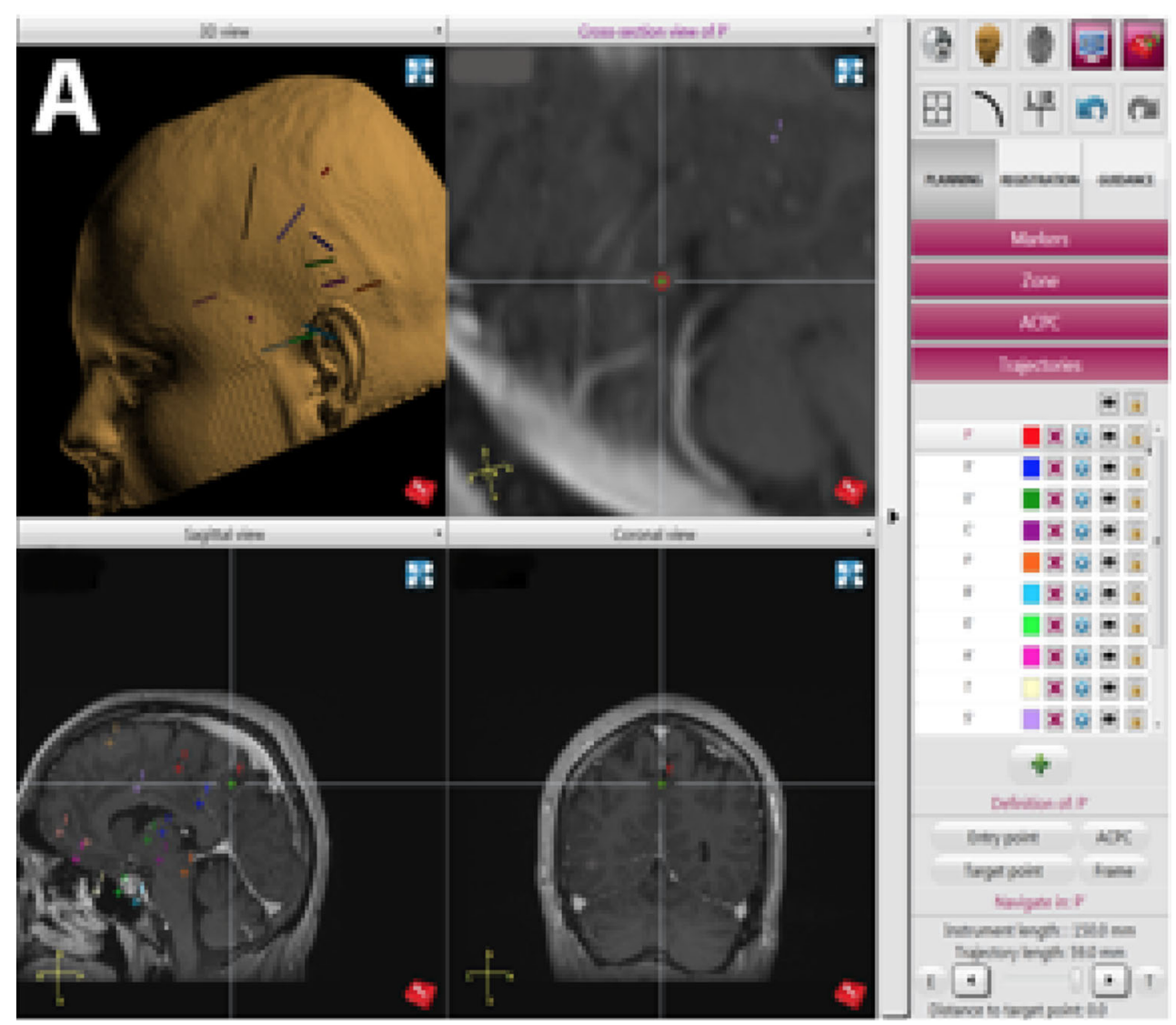

practice in cognitive studies of language production (Bock 1996, p. 407), participants were first familiarized with the pictures; they named them one by one and received oral corrective feedback when they provided unexpected responses, in order to reduce the diversity of possible responses. During the experimental testing itself, participants were instructed to name each object as fast as possible and to remain silent if they did not recognize the picture or could not name it. The pictures in a block were either from a single semantic category (e.g., vehicles; semantically homogeneous block) or from the 6 different semantic categories (semantically heterogeneous block). No attempt was made to analyze the blocks separately (as done by Ewald et al. 2012) in the current study, which remained focused on the task network rather than its modulations.

The experiment was controlled by the software E-Prime v2.0.1 (Psychology Software Tools, Pittsburgh, USA). For the duration of the procedure, an experimenter was sitting near the participant to monitor the task and take note of erroneous responses. In four participants, naming
Table 2 Demographic and clinical data information for each patient

\begin{tabular}{lllllll}
\hline ID & Age [yr.] & Sex & Hand. & LDH & DOE [yr.] & CEZ \\
\hline 1 & 35 & female & right & left & 23 & L MTL \\
2 & 45 & male & right & N/A & 44 & R MTL \\
3 & 61 & female & right & left & 11 & L TSR \\
4 & 53 & female & right & left & 18 & R FTL \\
5 & 48 & female & left & left & 4 & L MTL \\
6 & 27 & male & right & left & 19 & L TpsR \\
7 & 40 & female & right & left & 23 & L MTL \\
8 & 31 & male & right & left & 2 & L TPR \\
\hline
\end{tabular}

Hand., Handedness; LDH, Language-Dominant Hemisphere; DOE, Duration Of Epilepsy; CEZ, Clinicallyannotated Epileptogenic Zone; MTL, Mesial Temporal Lobe; FTL, Fronto Temporal Lobe; TSR, Temporosylvian Region; TpsR, Temporo-perisylvian Region; TPR, Temporo-parietal Region; L, Left; R, Right 


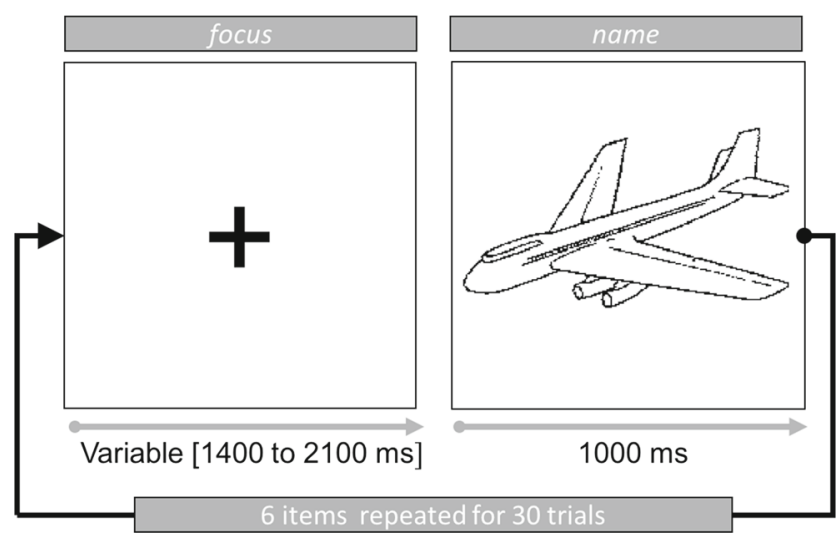

Fig. 3 Trial structure in the experimental task. Patients named pictures in various blocks of 30 trials each, constructed by repeating 6 different items in a random order. The pictures were from Snodgrass and Vanderwart (1980)

latencies were recorded with a microphone. When available (i.e., for half the patients), these latencies were used to identify inappropriate recordings, defined as trials with a response time inferior to $200 \mathrm{~ms}$ (e.g., anticipations or noisy hesitations). In all participants, inappropriate responses (errors, omissions, etc.) were identified online by the experimenter during the procedure. All inappropriate recordings and responses were excluded from the functional data analysis.

\subsection{Neural recordings}

During the task, continuous SEEG was recorded for all participants. For each participant, 8-13 stereotactically placed depth electrodes were implanted. The electrode contacts were $0.8 \mathrm{~mm}$ in diameter, $2 \mathrm{~mm}$ in length, and spaced $1.5 \mathrm{~mm}$ apart. Depth electrodes were inserted in either orthogonal or oblique orientations using a robotic surgical implantation platform (ROSA, Medtech Surgical Inc., USA) allowing intracranial recording from lateral, intermediate and/or deep cortical and subcortical structures in a three-dimensional arrangement (Johnson et al. 2014). The day prior to surgery, volumetric preoperative MRIs (T1, contrasted with Multihance $0.1 \mathrm{mmol}$ $\mathrm{kg}^{-1}$ ) were obtained and used to pre-operatively plan electrode trajectories. All trajectories were evaluated for safety; any trajectory that appeared to compromise vascular structures was adjusted appropriately without affecting the sampling from areas of interest.

SEEG electrophysiological data was acquired using a conventional clinical electrophysiology acquisition system (Nihon Kohden 1200, Nihon Kohden America, USA) at a sampling rate of $1 \mathrm{kHz}$ and $300 \mathrm{~Hz}$ anti-aliasing filter. Behavioral event data were simultaneously acquired during behavioral experiments along with the SEEG electrophysiology and stored for subsequent analysis. All signals were referenced to a contact affixed to the skull. Archived electrophysiological data was not filtered prior to offline analysis.

Each patient had electrode contacts characterized according to anatomical location. The anatomical locations of all contacts were identified through inspection of post-operative imaging, requiring agreement by two clinical experts.

\subsection{Regions of interest}

Ten anatomical regions of interest (ROIs) were selected out of the 83 regions available based on previous work on the word production network reviewed in the introduction. These ROIs comprised four regions of the left temporal lobe, two regions of the left parietal lobe, and four regions
Fig. 4 Anatomical sampling in the study. The sampling covers a substantial part of the regions involved in word production. Different arbitrary colors are used to highlight different regions. The numbers indicate contact and patient sampling in each region (number of contacts/patients). The abbreviations are as in Table 3

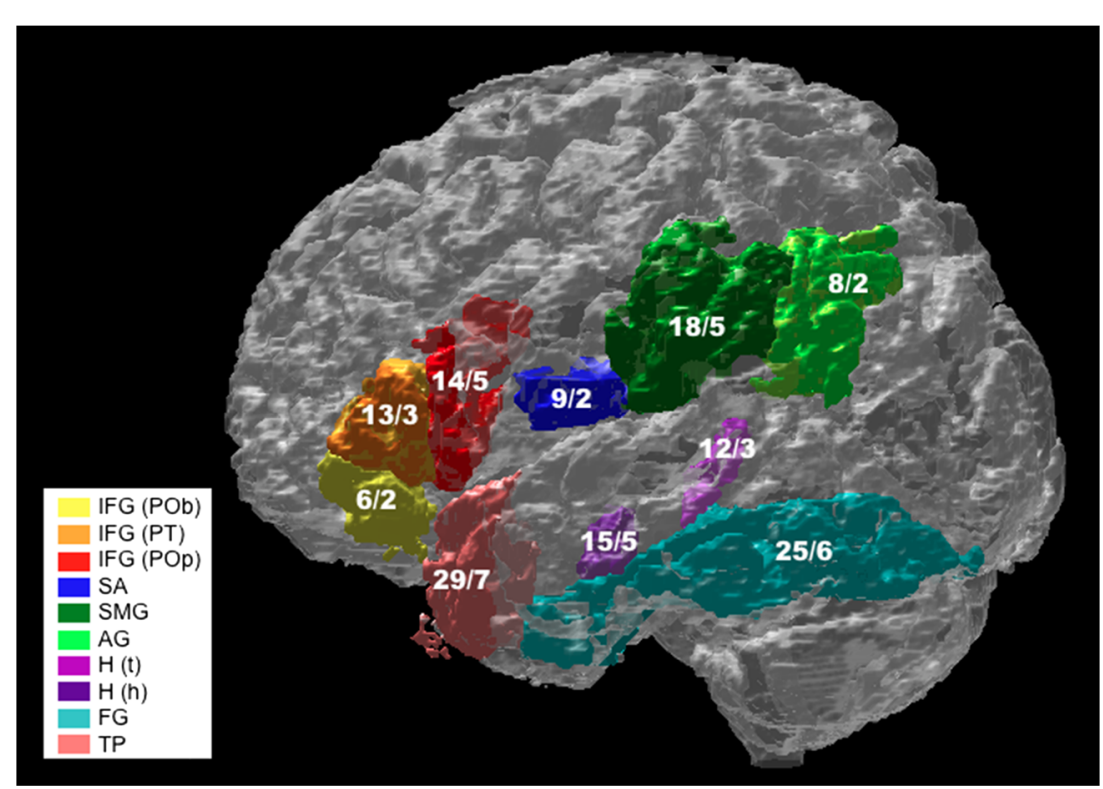


Table 3 Left hemisphere coverage in each patient

\begin{tabular}{llll}
\hline ID & Temporal & Parietal & Frontal \\
\hline 1 & FG, H (h), H (t), TP & AG, SMG & IFG (POp) \\
2 & TP & SMG & IFG (POb,POp) \\
3 & FG, H (t) & AG, SMG & IFG (POp) \\
4 & FG, H (h), TP & - & IFG (POp,PT) \\
5 & FG, H (h), TP & SMG & SA \\
6 & TP & - & IFG (POb,PT) \\
7 & FG, H (h/t), TP & SMG & IFG (PT) \\
8 & FG, H (h), TP & - & IFG (POp), SA \\
\hline
\end{tabular}

AG, Angular Gyrus; FG, Fusiform Gyrus; H (h), Hippocampus (Head); H (t), Hippocampus (Tail); IFG (POb), Inferior Frontal Gyrus (Pars Orbitalis); IFG (POp), Inferior Frontal Gyrus (Pars Opercularis); IFG (PT), Inferior Frontal Gyrus (Pars Triangularis); SA, Subcentral Area; SMG, Supramarginal Gyrus; TP, Temporal Pole

of the left frontal lobe, which are illustrated in Fig. 4 and detailed in Table 3. Even if none of the participants had recordings from all ten regions, it was checked that each one had at least one recording in the posterior temporal/parietal region or in the frontal zone, to provide for a sizable connectivity analysis.

\subsection{Signal processing}

Spectral analysis, artifact removal, and normalization To remove electric line noise, frequencies around $60 \mathrm{~Hz}$ and their harmonics were attenuated using a Notch filter, specifically, a second-order IIR notch filter centered around $60 \mathrm{~Hz}$ with a bandwidth of $3 \mathrm{~Hz}$ and a bandwidth attenuation of $1 \mathrm{~dB}$; the same parameters were then used to remove the first harmonic, i.e. centered around $120 \mathrm{~Hz}$ ). The oscillatory power of each signal was then calculated using the Continuous Morlet Wavelet Transform (CWT) (Morlet 1983). Wavelet coefficients were calculated for each SEEG electrode contact at 33 different frequencies ranging from 10 to $155 \mathrm{~Hz}$. Wavelet power time-series were computed by taking the square absolute value of these frequency coefficients, and smoothing them in time using a $100 \mathrm{~ms}$ window sliding at $25 \mathrm{~ms}$ steps. The wavelet method was chosen over the usual Fourier transform (Dumermuth et al. 1977) to preserve the time-course information of the SEEG signal. However, it is noteworthy that the temporal width of the wavelet varies as a function of the frequency (Herrmann et al. 2005). The duration of the sliding window $(100 \mathrm{~ms})$ was selected to cover the entire width of the wider wavelet (i.e., the $10 \mathrm{~Hz}$ wavelet) which was of approximately $95 \mathrm{~ms}$.

Extreme values of power, potentially related to artifacts, were identified and treated as follows. For every contact, the $\log$ of the power was computed for each of the 33 Morlet frequencies separately, to take into account the $1 / f$ distribution of power across $f$. Within each frequency, log-power values were sorted and divided in 100 percentiles. Values above and below $1.5 \times I Q R(I Q R$ : interquartile range) were considered as outliers and excluded from the data. Later, after epoching (see below), trials were inspected for their proportions of such extreme power values. Trials in which at least 10 frequencies showed many extreme power values, defined as $75 \%$ or more excluded values, were removed from the analysis. This resulted in the exclusion of an average of $7.5 \pm 5.9$ trials per patient. Finally, the log-power time-series for each Morlet frequency was normalized by removing the mean and by dividing by the standard deviation of the pre-processed data.

Cluster-based nonparametric statistical test The next step of the analysis sought to identify contacts in the ROIs for which neural activity was significantly different between the baseline and the stimulus periods, i.e., which contacts contained task-relevant information. To do so, the normalized signal (see previous section) was segmented into $1500 \mathrm{~ms}$ epochs containing the fixation point and the picture display for every trial. Each of these epochs was further separated in two distinct periods to be compared: the baseline period and the stimulus-response period.

Baseline periods were time-locked to the onset of the fixation point and lasted $500 \mathrm{~ms}$. Stimulus-response periods were time-locked to the onset of the picture and lasted $1000 \mathrm{~ms}$. Because baseline periods were half the duration of picture periods (21 time-points vs. 41 ), the mean value of the log power across the 21 time windows was computed and replicated to reach a total duration of 41 time windows (i.e., baseline periods were represented by their log-power average across time). This choice was made after verifying that the baseline appeared rather stable across time for the whole duration of each epoch, with minimal deviations from the mean $(S D /$ mean $\simeq 0.0015)$.

The statistical analysis explored the contrast between baseline vs. stimulus periods across all time-points and frequency values using independent two-sample $t$-tests. To deal with the multiple comparisons problem across time and frequency, a cluster-based non-parametric statistical test was used (Maris and Oostenveld 2007; Maris 2012), adapted here for intracerebral data. For each contact, a surrogate distribution of cluster values was created by permuting the condition labels (i.e., baseline vs. stimulus period) between trials. Within each of the 2000 random partition, $t$-statistics were computed for each time and frequency combination using an independent two-sample $t$-test. The resulting $p$-values were stored in a new $p$ value map $(2000 \times$ time $\times$ frequency). Significant samples ( $p$-value $<0.05)$ that were adjacent either in frequency or in time in this map were grouped in clusters. Clusterlevel statistics were calculated by taking the sum of all the $t$-statistics within each cluster; the maximum value 


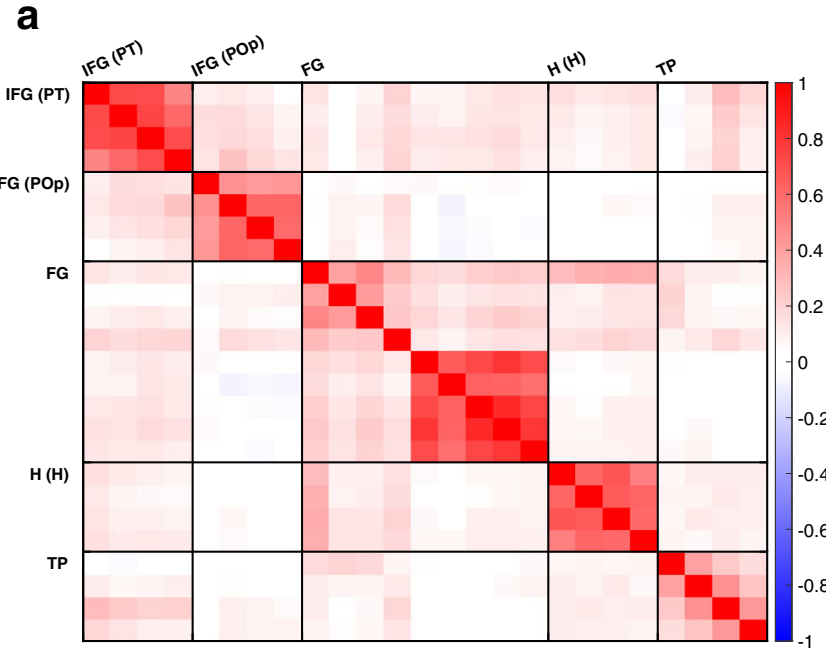

Fig. 5 Connectivity Analysis for the $\beta$ band of sample Participant 4. a Trial-by-trial Pearson Correlation Matrix. Red values (Pearson's correlation coefficient near 1) indicate nearly total positive linear correlation, white values (Pearson's correlation coefficient near 0) indicate no linear correlation, and blue values (Pearson's correlation coefficient near -1 ) is nearly total negative linear correlation. Regions

across the clusters of each permutation were included in the surrogate distribution. By putting the 2000 random partitions together to form a new null-distribution at the cluster-statistic level, it was now possible to compare the observed cluster-level statistics resulting from the real comparison between the two conditions and to compute their final cluster-statistic significance $(p$-value $<0.05)$. With this method, effects that are strong, long-lasting, and/or involving several frequencies are more likely to be significant than localized transient effects. Contacts in which no significant cluster was found were removed from further analysis.

The statistical clustering procedure was computed for the whole time-frequency plane, which was then inspected in more detail. Based on this examination, and on our interest for the $\beta$ and the $\gamma_{\text {high }}$ band (see Introduction), the significant clusters were cropped to their intersection with the frequency bands of interest: the $\beta$ band $(12-30 \mathrm{~Hz})$, and the $\gamma_{\text {high }}$ band $(70-150 \mathrm{~Hz})$.

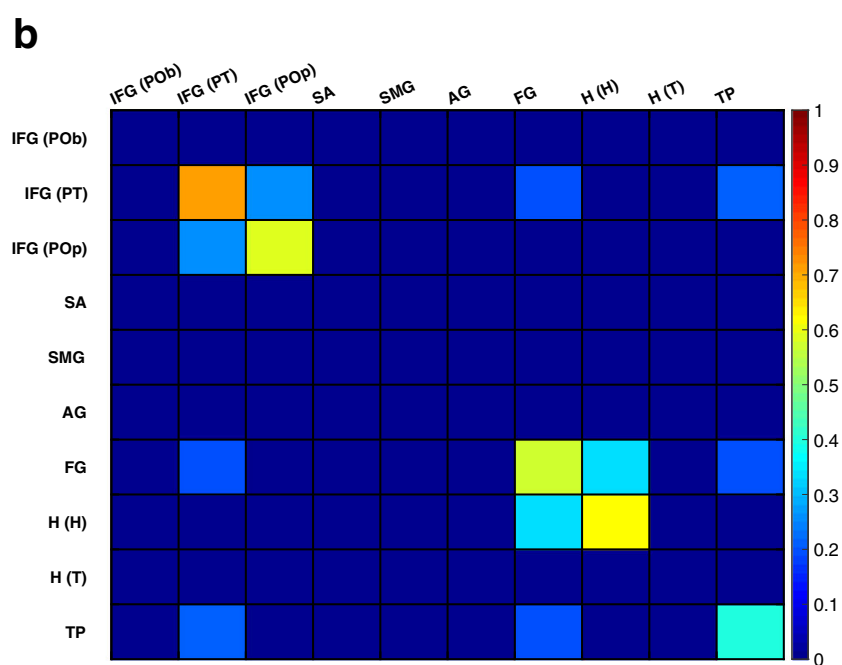

sampled in this patient, abbreviations as in Table 3. b Summarized Pearson Correlation Matrix. The Pearson's correlation coefficients have been summarized across contacts within each region, see text for details on the procedure. Regions sampled in all patients, abbreviations as in Table 3

\subsection{Connectivity analysis}

The operationalization of the hypothesis to be tested was that two brain regions are functionally connected if their trial-by-trial correlation in a specified frequency band was significantly positive or negative when compared to a nulldistribution.

Within each patient To test the hypothesis, we first computed two signals for each task-relevant contact and for each trial: $\beta$ power and $\gamma_{\text {high }}$ power timeseries. Then, for every task-relevant pair of contacts (i.e., with significant task-evoked activity), we computed the Pearson's correlation coefficient between the time-series of single trials within each frequency band. This correlation has a value of 1 for total positive linear correlation, 0 for no linear correlation, and -1 for total negative linear correlation (Fig. 5a). The inter-regions pairwise correlations form a distribution for which we computed the 5\% and $95 \%$
Table 4 Behavioral performance across the patients

\begin{tabular}{llll}
\hline ID & Number of trials & Correct / Other & Response time $[\mathrm{ms}](\mathrm{mean} \pm \mathrm{SD})$ \\
\hline 1 & 240 & $240 / 0$ & NA \\
2 & 240 & $240 / 0$ & NA \\
3 & 240 & $233 / 7$ & $849 \pm 229$ \\
4 & 240 & $212 / 28$ & $999 \pm 415$ \\
5 & 240 & $240 / 0$ & NA \\
6 & 120 & $120 / 0$ & NA \\
7 & 240 & $240 / 0$ & $753 \pm 179$ \\
8 & 180 & $153 / 27$ & $1137 \pm 381$ \\
\hline
\end{tabular}


confidence bounds. Every pair of task-relevant contacts that fell below the $5^{\text {th }}$ percentile or above the $95^{t h}$ percentile were designated as significantly functionally connected. Finally, we created a patient-specific connectivity matrix whose $(i, j)$ entry was the proportion of $(i, j)$ contacts-pairs that were both task-relevant and functionally connected above threshold, among all the $(i, j)$ contacts-pairs sampled in the patient (Fig. 5b).

Group analysis Beyond the quantification of intra-patient connectivity as single cases, a group analysis was computed to derive a more general picture of the word production network while dealing with the inevitable variability of brain signal across patients. Specifically, the binary (thresholded) connectivity matrices for each frequency band were added and a percentage matrix was computed by dividing each summed number by the actual total number of available connectivities.

\section{Results}

\subsection{Behavioral results}

A summary of behavioral performance across participants can be found in Table 4; when available, naming latencies are included. The rate of correct response was fairly good compared to other patients of the same population. As expected, the available naming latencies appeared highly variable from trial to trial within the $600-1400 \mathrm{~ms}$ range, a range which is typical also of healthy speakers (Snodgrass and Yuditsky 1996). Based on these values, a window duration of $1000 \mathrm{~ms}$ following picture onset was hypothesized to encompass most of the language production processes across participants and trials. The signal processing procedure described above (e.g. definition of time-window of interest) took into account this observation and was applied to correct trials only.

\subsection{Task activity}

The satellite map (Fig. 6) displays an overview of the activities elicited by the task in the frequency domain, for all brain areas of interest, across all patients. Regarding this task activity, the most remarkable result is the high consistency within and across patients of the responses observed in FG: a positive cluster with an early latency in higher frequencies, and a negative cluster in lower frequencies. A comparable pattern, delayed in latency, is observed in IFG Pars Opercularis, although here high frequency negative clusters prevail on some contacts. A somewhat similar pattern of activity, but much more variable across contacts and patients, was detected in the other sub-areas of IFG, Pars Triangularis and Pars Orbitalis, as well as in the parietal ROIs SMG and AG. SA activation, when detected, is clearly present later, around the time where vocalization begins ( $700-850 \mathrm{~ms})$. This late response is observed in three contacts out of seven, all in one patient, as would be expected if the activation where very focal. Finally, between one third and one half of the contacts in Hippocampus (head and tail) and TP showed positive or negative clusters in high frequencies. Most of the clusters, however, were observed in low frequencies. There was great variability of the activation pattern within these latter ROIs. This could be expected for the large definition of region TP, and was more surprising for the much smaller sub-regions within hippocampus. Again, this is suggestive of rather focal responses, not detected on all contacts.

As a complementary information, the cells on the diagonal of the $\beta$ and $\gamma_{\text {high percentage matrices on }}$ Fig. 7 summarize within-patient homogeneity for each region or, more specifically, above-threshold withinpatient correlations between the different recording contacts in each region. Within-patient consistency was always high, with the possible exception of IFG Pars Orbitalis.

\subsection{Functional connectivity results}

Group connectivity matrices (Fig. 7) quantify the similarity of task related neurophysiological activity across the brain areas of interest, now at the level of the population studied. These matrices indicate, for each pair of regions, the proportion of patients showing supra-threshold connectivity, based on the single-trial correlation of activities between the two regions (see Methods for details). Such proportion is always computed against the sub-population for which each pair of regions was simultaneously sampled (i.e., between 1 and 5 patients; sampling details in Table 3). The matrices on Fig. 7 are further summarized in Fig. 8.

The most visible observation is that, within the sublobar frontal, temporal, and parietal regions (Table 3), connectivity was by and large similarly detected in the $\gamma_{\text {high }}$ and $\beta$ bands. Between lobe connectivity is much more discrete. Parieto-frontal and fronto-temporal network connectivity are more often detected in $\gamma_{\text {high }}$ than in $\beta$ band, while the parieto-temporal network reveals largely similar connections in the two frequency bands. We review in turn each of these nodes, and then each of the sub-networks.

Frontal regions There were significant correlations between all three IFG regions, although such pairs were sampled only on one patient each. SA showed no correlation IFG Pars Opercularis. 


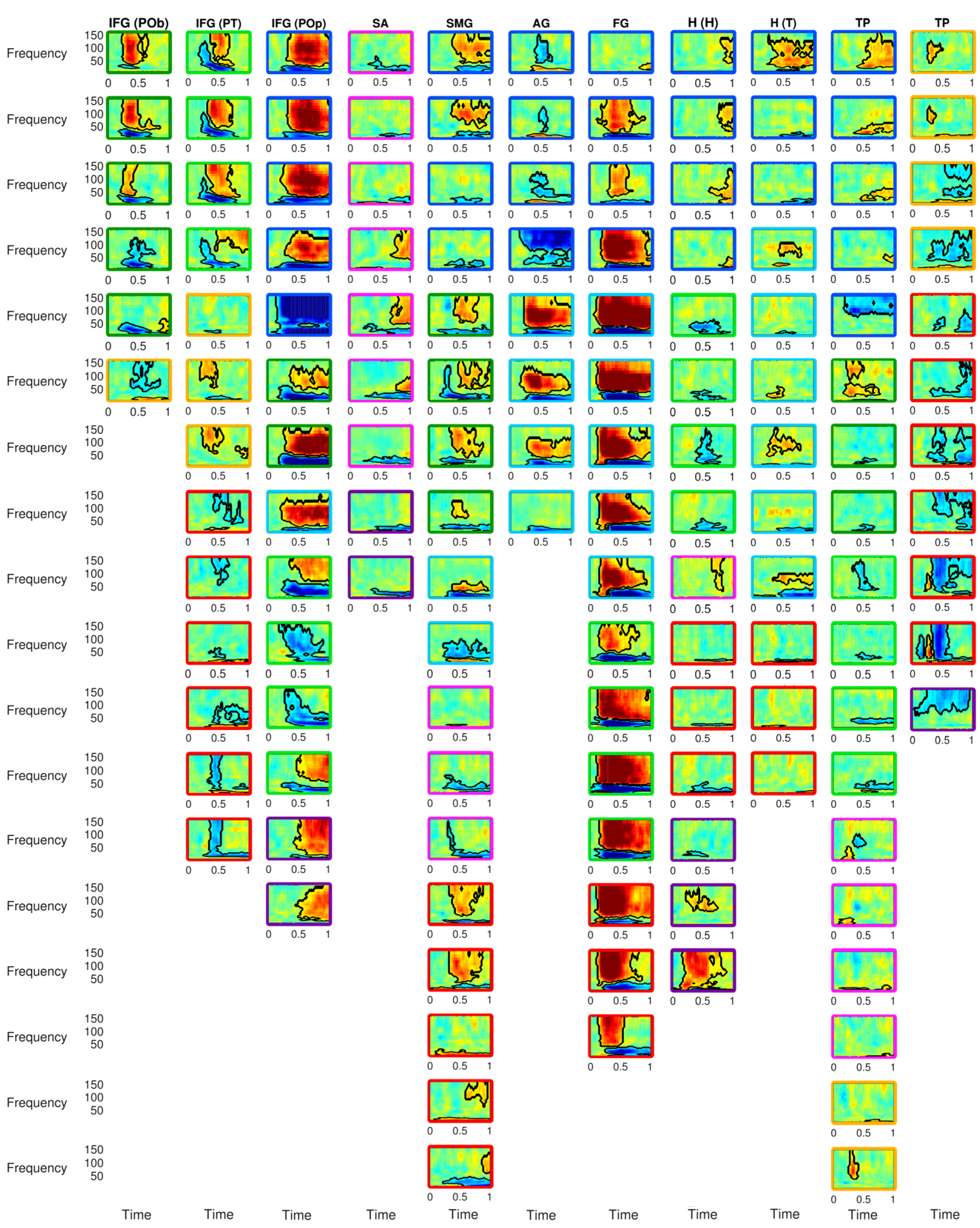

Fig. 6 Overview of task activity on all the recording contacts. Each column represents a region. Each panel represents a bi-polar recording montage (difference between adjacent contacts), and depicts the baseline corrected power across the frequency plane $(0-150 \mathrm{~Hz})$,

during the stimulus-response interval $(0-1 \mathrm{~s})$. The time-frequency clusters circled in black were revealed by the non-parametric significance test. The color border of each panel codes patient identity 


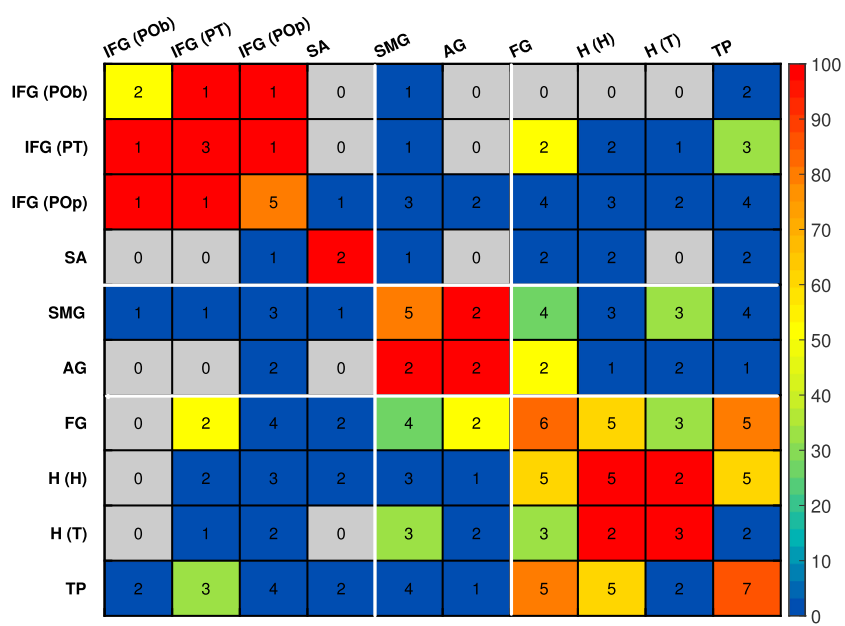

(a) $\beta$ Percentage Matrix.

Fig. 7 Whole Population Connectivity Analysis in the Picture Naming Task, Distinguishing the $\beta$ and $\gamma_{\text {high }}$ Bands. The colour scale indicates the percentage of patients with supra-threshold connectivity between each pair of ROIs. The number in each block indicates the total
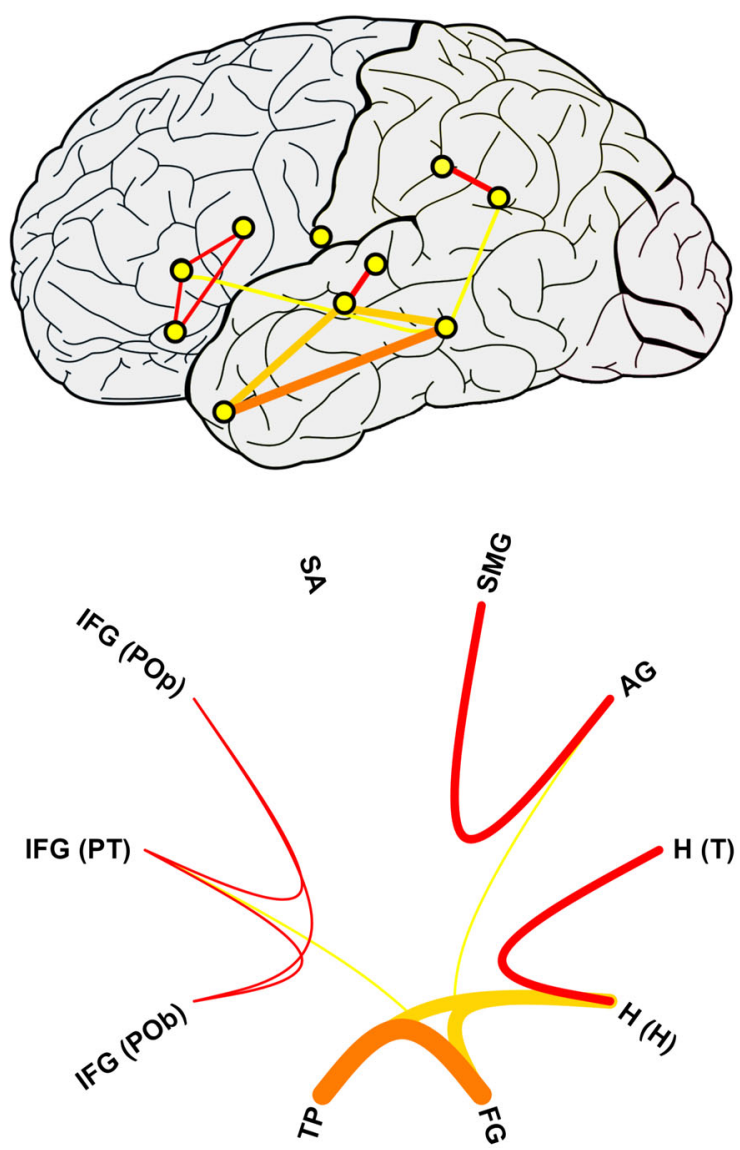

Fig. 8 Summary of the functional connectivity observed during word production across two frequency bands. The left and right columns are for $\beta$ and $\gamma_{\text {high }}$ frequency bands, respectively. (Top) Schematic representation on a saggital view of the left hemisphere of the functional connections between the ten regions of interest. (Bottom) Network representation of the same data. Color represents the proportion of patients showing supra-threshold connectivity, as in Fig. 7. Line

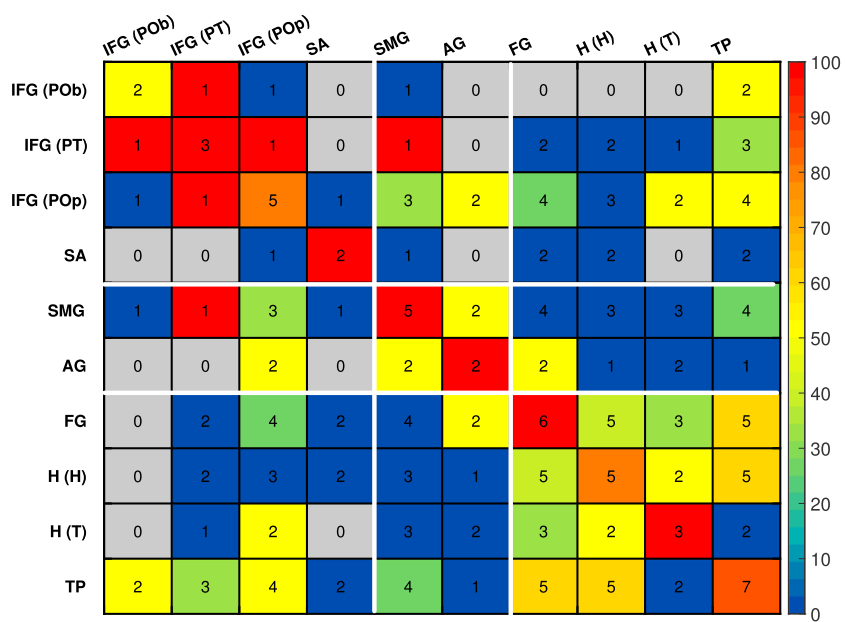

(b) $\gamma_{\text {high }}$ Percentage Matrix.

number of participants in which the pair of ROIs was sampled. Grey cells correspond to pairs of regions that were not sampled simultaneously within any patient. Region name abbreviations are as in Table 3

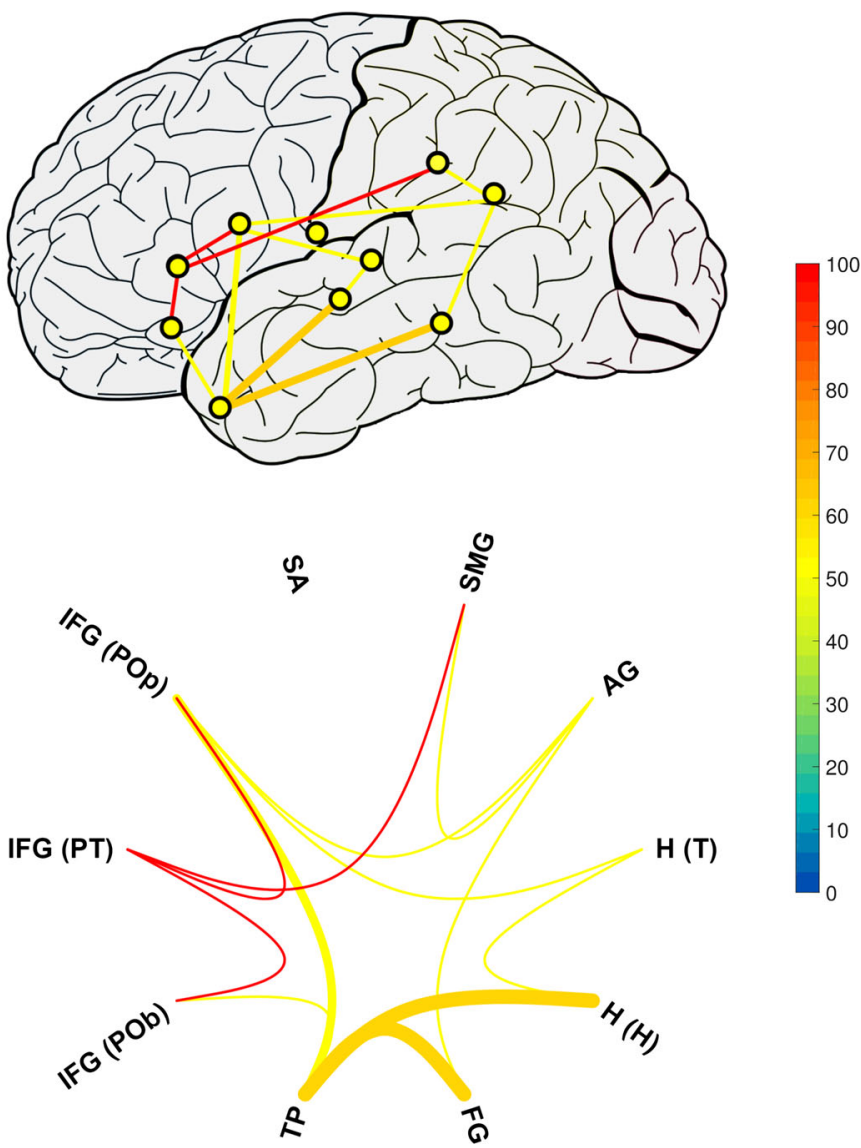

thickness represents the number of patients in the sample. Region abbreviations are for: AG, Angular Gyrus; FG, Fusiform Gyrus; $\mathrm{H}$ (h), Hippocampus (head); H (t), Hippocampus (tail); IFG (POb), Inferior Frontal Gyrus (Pars Orbitalis); IFG (POp), Inferior Frontal Gyrus (Pars Opercularis); IFG (PT), Inferior Frontal Gyrus (Pars Triangularis); SA, Subcentral Area; SMG, Supramarginal Gyrus; TP, Temporal Pole 
Parietal regions The two regions were consistently connected (in 2 out of 2 patients) in the $\beta$ band, but less consistently so ( 1 out of 2 patients) in the $\gamma_{\text {high }}$ band.

Temporal regions Among temporal regions, the clearest functional connectivity was observed within hippocampus, between its head and tail, in the $\beta$ band (and for 1 out of 2 patients in the $\gamma_{\text {high }}$ band). There were reliable connections between FG, TP, and the head of hippocampus. More specifically, FG and TP activity were correlated in the $\beta$ band, and slightly less reliably in the $\gamma_{\text {high }}$ band; FG and the head of hippocampus also showed correlated activity, again more reliable in the $\beta$ band; finally, TP and the head of hippocampus were correlated in both frequency bands. We note that temporal regions were those that were most often sampled in this studied (higher patient numbers for each region, visible in the diagonal cells), hence providing the more robust test within the ventral pathway.

Parieto-frontal network SMG was connected with IFG Pars Triangularis in the $\gamma_{\text {high }}$ band, more reliably than to IFG Pars Opercularis. AG, in turn, was connected (in 1 out of 2 patients) to IFG Pars Opercularis. The other connections, particularly in $\beta$, were either not-significant or not sampled.

Parieto-temporal network Connectivity was observed (in 1 out of 2 patients) between AG and FG in both frequency bands. The connections between SMG and temporal regions were less reliable.

Fronto-temporal network The fronto-temporal network is visible in a subset of the patients, never more than 2 out of 4. IFG Pars Triangularis and FG were correlated in the $\beta$ band, but not in the $\gamma_{\text {high }}$ band. IFG Pars Opercularis was correlated to TP and the tail of hippocampus, both in the $\gamma_{\text {high }}$ band. Connectivity with Pars Orbitalis could only be assessed with the temporal pole (TP) and was present in 1 out of 2 patients. SA did not show any consistent connectivity with the temporal regions.

\section{General discussion}

Previous research has established the existence of various pathways connecting language regions, often on the basis of animal models, or human functional connectivity at rest. Only a few studies have resorted to neurophysiological signals recorded during word production tasks have also been analyzed. These have used a variety of processing methods, and highlighted quite diverse conclusions (Table 1). Given this diversity, more research is needed to understand the functional connectivity underlying the primary ability of word production.
In the current study, we explored whether the posited language processing pathways could be identified in neurophysiological signals during a cognitive language production task. We resorted to bipolar stereo-tactic EEG (SEEG) measures, allowing a very precise location of activities recorded with high temporal resolution. We combined a cluster-based non-parametric statistical test, to identify task-active regions, with a frequency-specific correlation measure, to assess functional connectivity between recording contacts across regions. Our exploration was focused on frontal, parietal and temporal regions, which have been previously associated in various models with semantically driven word production (e.g. during picture naming: Hickok 2012; Roelofs 2014; Ueno et al. 2011).

\subsection{Minor differences in the $\beta$ and $\gamma_{\text {high }}$ networks}

In language research, particularly about language production, the specific roles played by oscillations occurring at different frequencies remain largely an open question. The $\beta$ and $\gamma_{\text {high }}$ bands were distinguished here because they have been linked to various language production processes in different contexts (see Introduction).

Based on current frameworks (Donner and Siegel 2011), we expected that lower frequency oscillations would play a prominent role in long-range communication and information transfer. Surprisingly, this was not the case. A possible interpretation of the regional (i.e., within lobe) $\beta$ network we observed would be a better efficiency of top-down modulation conveyed by the $\beta$ rhythms (Engel and Fries 2010). Overt picture naming task requires the integration of lexico-semantic, and phonological information, via selection processes, which can be linked to the temporal, parietal and frontal lobes, respectively (Indefrey 2011). A reasonable speculation is that the local connectivity in the beta band within these regions reflects the dominance of endogenous top-down influences for a faster and accurate response. Because the connectivity in both frequency bands was not markedly different, we will not systematically distinguish $\beta$ and $\gamma_{\text {high }}$ connectivity in the discussion, as originally planned, only acknowledge that the findings more often concerned $\gamma_{\text {high }}$. Still, given the postulated contrast between the roles of the two frequency bands inter-areal communication, it would be important that future work on functional connectivity during word production explores neural activation beyond the more popular $\gamma_{\text {high }}$ band (e.g., Llorens et al. 2016; Piai et al. 2015).

\subsection{Functional sub-networks revealed}

Parieto-frontal network Regions in the left parietal lobe (AG and SMG) were connected in some patients to different sub-regions in the frontal area. This is in broad 
agreement with previous findings in structural connectivity (Catani and Mesulam 2008; Petrides 2014), as well as resting state functional connectivity (Margulies and Petrides 2013; Parlatini et al. 2017). These regions are linked by the superior longitudinal fasciculus and the arcuate fasciculus, whose damage entails conduction aphasia. We note however that our results did not reveal more fine grained distinctions that could have been expected (Margulies and Petrides 2013; see also Petrides and Pandya 2009). For example, we expected that SMG would show preferential connectivity to Pars opercularis, but instead we observed more reliable connectivity to Pars Triangularis. In contrast, Pars Opercularis was significantly connected to AG in one patient. The exploratory nature of our analysis, particularly the thresholding procedure, warrants further assessments of the differential strengths of the connections in these contrasts. We come back to this point below.

Parieto-temporal network We highlight the connectivity between both parietal regions (supra-marginal gyrus, SMG, and angular gyrus, AG), and the fusiform gyrus FG. The AG-FG connection is consistent with Uddin et al. (2010), and could presumably be sustained by the temporo-parietal white matter bundle connecting these regions ( $\mathrm{Wu}$ et al. 2016).

Fronto-temporal network Significant functional connectivity was detected in some patients between the temporal pole and the three IFG subdivisions. These regions are anatomically connected by the uncinate fasciculus and the extreme capsule Catani et al. (2005). The importance of this ventral pathway for semantically driven word production has been debated, with arguments in favor (Ueno et al. 2011) and against it (Roelofs 2014). While these results do not establish the direct connectivity, let alone anatomical connections, they give some support to the model in which semantically driven word production does involve a ventral pathway (Ueno et al. 2011). This conclusion does not exclude the possibility of the dorsal pathway mediated by AG, but we did not detect here any significant AG-TP connectivity (sampled in one patient).

The IFG has been frequently attributed roles in selection processes and in formulating an articulatory code during word production (Indefrey 2011; for evidence based on intra-cranial $\gamma_{\text {high }}$, see: Flinker et al. 2015, Riès et al. 2017). Consistent with a hierarchical structure of functions across the three sub-parts of IFG (see Bourguignon 2014, for review) in IFG, the significant connectivity between temporal areas and IFG was detected most often with Pars Opercularis in our picture naming task. The hippocampus tail displayed reliable connectivity with Pars Opercularis in 1 out of 2 patients. If confirmed, such hippocampal-prefrontal functional connectivity during word retrieval would add some credence to the involvement of hippocampus in visual word retrieval (Hamamé et al. 2014; Llorens et al. 2016; see also Covington and Duff 2016; Piai et al. 2016; Sheldon et al. 2016).

In contrast, we did not observe any significant connectivity between Pars Opercularis and SA, which is part of the ventral sensory motor cortex (Conant et al. 2018; Conant et al. for an earlier review, see 2014). This is in contrast with the reliable $\gamma_{\text {high }}$ functional connectivity, quantified with the Granger causality metric, reported by Flinker et al. (2015) between "Broca's" and motor areas. The connectivity we used is not time-resolved, and is computed with zero-lag, therefore it only reveals similar simultaneous activation across regions (see next section).

\subsection{The choice of a connectivity metric}

The connectivity metric we chose, correlation in the time-frequency domain for two frequency bands $(\beta$ and $\gamma_{\text {high}}$ ), was intended to reveal zero-lag similarity between regions irrespective of their signal power. Zerolag similarity captures, in broad lines, the cognitive hypothesis of simultaneous (parallel) processing (Dubarry et al. 2017). While serving this purpose, the metric likely misses other relevant signal information. Beyond the choice of a particular metric, the analysis also required deciding about significance thresholds and summarizing procedures for the group analysis. Clearly, some of these decisions were heuristic, and the choices made were rather conservative (95\% threshold for the correlations). It would be appropriate, in future studies, to perform a more exhaustive exploration of the connectivity space, contrasting various metrics and thresholds (as in Wang et al. 2014). This exploration should be guided by a conjunction of cognitive and neurophysiological principles regarding the different processes that conform word production (Friederici and Singer 2015).

\subsection{Limitations}

Our study has the usual limitations inherent to studies of intracerebral activity in epileptic patients (reviewed in detail in Lachaux et al. 2012). While there was a fairly diverse spatial sampling across the patients, some key regions were not available (e.g. middle temporal gyrus, often linked to lexical processing). In terms of numbers, a fair count of patients was available for every ROI, although the counts for ROI pairs was inevitably inferior. Overall, 9 out of 55 ROI pairs were not sampled (16\%), but we note that 36 of them were sampled in two patients or more $(65 \%)$. More thorough investigations will certainly be needed, perhaps focusing on each pathway at a time, with larger number of patients for each ROI pair. 
The limitations in spatial sampling were partially compensated by our classification of intra-cerebral contacts into broad regions, at the expense of the highlyspecific anatomical details that are characteristic of each patient's stereotactic implantation coordinates. This spatial smoothing does not allow exploring finer grained subdivisions within each region. Indeed, some of the inconsistencies between our data and the literature based on other methods might be resolved in the future by finer-grained anatomical classification of the patients' contacts.

As noted in the previous section, the signal processing procedure included a number of arbitrary decisions (e.g. thresholding step for quantifying the functional connectivity measure at the group level). This part of the procedure was exploratory, and we did not assess the impact of such thresholding decisions beyond some informal verifications.

\subsection{Conclusion and perspectives}

The neural regions recruited during word production are relatively well established. Despite their recent framing in network and processing stream models, their functional connectivity has rarely been explored during language production tasks. In the current study, we started with neurophysiological signal with the highest temporal and spatial resolution, sacrificed some of its temporal resolution (time-frequency transformation, connectivity estimates in 1 second long windows), and of its spatial resolution (grouping of contacts in broad meaningful regions) in the interest of a group level analysis quantifying similar simultaneous activity across regions.

The results demonstrate some properties of three functional sub-networks, between the areas sampled in the temporal, parietal, and frontal lobes. Unexpectedly, longrange connectivity patterns were relatively similar in the $\beta$ and $\gamma_{\text {high }}$ bands, possibly even more reliable in the $\gamma_{\text {high }}$ band. The observed functional connectivities were by and large consistent with white matter tracts postulated for the language circuitry. Among the main observations was the functional connectivity between the temporal pole and the inferior frontal gyrus, compatible with a ventral processing, as well as the links observed in some patients between parietal regions (SMG and $\mathrm{AG}$ ) and Pars Opercularis and Pars Triangularis, compatible with a dorsal processing pathway (but not in the details).

We believe that future work on task related functional connectivity during word production will benefit from signal processing developments (e.g., exploring other metrics integrating directionality or temporal lags, exploring significance threshold definitions), and from the spatial specificity of single-case explorations.

\subsection{On-line supplementary materials}

The code files used in this study can be downloaded from https://osf.io/8x9cy/.

Acknowledgments Research supported by grants ANR-11-LABX0036 (BLRI), ANR-16-CONV-0002 (ILCB), by the Excellence Initiative of Aix-Marseille University (A*MIDEX), and by a Kavli Neuroscience Discovery Institute postdoctoral fellowship awarded to P.S.

\section{Compliance with Ethical Standards}

Conflict of interests The authors declare that they have no conflict of interest.

\section{References}

Binder, J.R., \& Desai, R.H. (2011). The neurobiology of semantic memory. Trends in Cognitive Sciences, 15(11), 527-536. https:// doi.org/10.1016/j.tics.2011.10.001.

Bock, K. (1996). Language production: methods and methodologies. Psychonomic Bulletin \& Review, 3(4), 395-421. https://doi.org/ $10.3758 / \mathrm{BF} 03214545$.

Bourguignon, N.J. (2014). A rostro-caudal axis for language in the frontal lobe: the role of executive control in speech production. Neuroscience and Biobehavioral Reviews, 47, 431444. https://doi.org/10.1016/j.neubiorev.2014.09.008.

Broca, P. (1861). Remarques sur le siège de la faculté du langage articulé, suivies d'une observation d'aphémie (perte de la parole) [Remarks on the seat of the faculty of articulated language, followed by an observation of aphemia (loss of speech)]. Bulletin et Mémoires de la Société Anatomique de Paris, 6, 330-357. http:// psychclassics.yorku.ca/Broca/aphemie-e.htm.

Catani, M., \& Mesulam, M. (2008). The arcuate fasciculus and the disconnection theme in language and aphasia: history and current state. Cortex, 44(8), 953-961. https://doi.org/10.1016/j. cortex.2008.04.002.

Catani, M., Jones, D.K., et al. (2005). Perisylvian language networks of the human brain. Annals of Neurology, 57(1), 8-16.

Clarke, A., \& Tyler, L.K. (2015). Understanding what we see: how we derive meaning from vision. Trends in Cognitive Sciences, 19(11), 677-687. https://doi.org/10.1016/j.tics.2015.08.008.

Collard, M.J., Fifer, M.S., Benz, H.L., McMullen, D.P., Wang, Y., Milsap, G.W., Korzeniewska, A., Crone, N.E. (2016). Cortical subnetwork dynamics during human language tasks. NeuroImage, 135, 261-272. https://doi.org/10.1016/j.neuroimage.2016.03.072.

Conant, D., Bouchard, K.E., Chang, E.F. (2014). Speech map in the human ventral sensory-motor cortex. Current Opinion in Neurobiology, 24, 63-67.

Conant, D.F., Bouchard, K.E., Leonard, M.K., Chang, E.F. (2018), Human sensorimotor cortex control of directly-measured vocal tract movements during vowel production. Journal of $\mathrm{Neu}$ roscience, 2382-17. https://doi.org/10.1523/JNEUROSCI.238217.2018.

Covington, N.V., \& Duff, M.C. (2016). Expanding the language network: direct contributions from the hippocampus. Trends in Cognitive Sciences, 20(12), 869-870. https://doi.org/10.1016/j.ti cs.2016.10.006.

Dell, G.S., Schwartz, M.F., Nozari, N., Faseyitan, O., Branch Coslett, H. (2013). Voxel-based lesion-parameter mapping: identifying the neural correlates of a computational model of word 
production. Cognition, 128(3), 380-396. https://doi.org/10.1016/ j.cognition.2013.05.007.

Donner, T.H., \& Siegel, M. (2011). A framework for local cortical oscillation patterns. Trends in Cognitive Sciences, 15(5), 191-199. https://doi.org/10.1016/j.tics.2011.03.007.

Dubarry, A.S., Llorens, A., Trèbuchon, A., Carron, R., LiègeoisChauvel, C., Bènar, C. G., Alario, F.X. (2017). Estimating parallel processing in a language task using single-trial intracerebral electroencephalography. Psychological Science, 28(4), 414-426. https://doi.org/10.1177/0956797616681296.

Dumermuth, G., Gasser, T., Germann, P., Hecker, A., Herdan, M., Lange, B. (1977). Studies on EEG activities in the beta band. European Neurology, 16(1-6), 197-202. https://doi.org/10.1159/ 000114900

Engel, A.K., \& Fries, P. (2010). Beta-band oscillations-signalling the status quo? Current Opinion in Neurobiology, 20(2), 156-165.

Ewald, A., Aristei, S., Nolte, G., Abdel-Rahman, R. (2012). Brain oscillations and functional connectivity during overt language production. Frontiers in Psychology, 3. https://doi.org/10.3389/ fpsyg.2012.00166.

Flinker, A., Korzeniewska, A., Shestyuk, A.Y., Franaszczuk, P.J., Dronkers, N.F., Knight, R.T., Crone, N.E. (2015). Redefining the role of Broca's area in speech. Proceedings of the National Academy of Sciences, 112(9), 2871-2875. https://doi.org/10.1073 /pnas.1414491112.

Fridriksson, J., Yourganov, G., Bonilha, L., Basilakos, A., Den Ouden, D.B., Rorden, C. (2016). Revealing the dual streams of speech processing. Proceedings of the National Academy of Sciences, 113(52), 15,108-15,113. https://doi.org/10.1073/pn as. 1614038114 .

Friederici, A.D., \& Singer, W. (2015). Grounding language processing on basic neurophysiological principles. Trends in Cognitive Sciences, 19(6), 1-10. https://doi.org/10.1016/j.tics.2015.03.012.

Fries, P. (2015). Rhythms for cognition: communication through coherence. Neuron, 88(1), 220-235. https://doi.org/10.1016/j.neu ron.2015.09.034.

Fuertinger, S., Horwitz, B., Simonyan, K. (2015). The functional connectome of speech control. PLOS Biology, 13(7), 1-31. https://doi.org/10.1371/journal.pbio.1002209.

Gaona, C.M., Sharma, M., Freudenburg, Z.V., Breshears, J.D., Bundy, D.T., Roland, J., Barbour, D.L., Schalk, G., Leuthardt, E.C. (2011). Nonuniform high-gamma $(60-500 \mathrm{~Hz})$ power changes dissociate cognitive task and anatomy in human cortex. The Journal of Neuroscience: the Official Journal of the Society for Neuroscience, 31(6), 2091-2100. https://doi.org/10.1523/JNE UROSCI.4722-10.2011.

Geschwind, N. (1970). The organization of language and the brain. Science, 170(3961), 940-944. https://doi.org/10.1126/scien ce.170.3961.940.

Goodale, M.A., \& Milner, A.D. (1992). Separate visual pathways for perception and action. Trends in Neurosciences, 15(1), 20-25. https://doi.org/10.1016/0166-2236(92)90344-8.

Grill-Spector, K., \& Weiner, K.S. (2014). The functional architecture of the ventral temporal cortex and its role in categorization. Nature Reviews Neuroscience, 15(8), 536-548. https://doi.org/10.1038/n rn3747.

Hamamė, C. M., Alario, F.X., Llorens, A., Liègeois-Chauvel, C., Trébuchon-Da, F.A. (2014). High frequency gamma activity in the left hippocampus predicts visual object naming performance. Brain and Language, 135, 104-114. https://doi.org/10.1016/j.ba ndl.2014.05.007.

Han, Z., Ma, Y., Gong, G., Huang, R., Song, L., Bi, Y. (2016). White matter pathway supporting phonological encoding in speech production: a multi-modal imaging study of brain damage patients. Brain Structure and Function, 221(1), 577-589. https://doi.org/10.1007/s00429-014-0926-2.

Hassan, M., Benquet, P., Biraben, A., Berrou, C., Dufor, O., Wendling, F. (2015). Dynamic reorganization of functional brain networks during picture naming. Cortex, 73, 276-288. https://doi.org/10.1016/j.cortex.2015.08.019.

Herrmann, C.S., Grigutsch, M., Busch, N.A. (2005). EEG oscillations and wavelet analysis. In Handy, T.C. (Ed.) Event-related potentials: a methods handbook (pp. 229-259). Cambridge: MIT Press.

Hickok, G. (2012). Computational neuroanatomy of speech production. Nature Reviews Neuroscience, 13, 135-45. https://doi.org/ 10.1038/nrn3158.

Indefrey, P. (2011). The spatial and temporal signatures of word production components: a critical update. Frontiers in Psychology, 2, 255. https://doi.org/10.3389/fpsyg.2011.00255.

Johnson, M.A., Thompson, S., Gonzalez-Martinez, J., Park, H.J., Bulacio, J., Najm, I., Kahn, K., Kerr, M., Sarma, S.V., Gale, J.T. (2014). Performing behavioral tasks in subjects with intracranial electrodes. Journal of Visualized Experiments: JoVE, 92, e51947. https://doi.org/10.3791/51947.

Korzeniewska, A., Franaszczuk, P.J., Crainiceanu, C.M., Kuł, R., Crone, N.E. (2011). Dynamics of large-scale cortical interactions at high gamma frequencies during word production: event related causality (ERC) analysis of human electrocorticography (ECoG). NeuroImage, 56(4), 2218-2237. https://doi.org/10.1016/ j.neuroimage.2011.03.030.

Lachaux, J.P., Axmacher, N., Mormann, F., Halgren, E., Crone, N.E. (2012). High-frequency neural activity and human cognition: past, present and possible future of intracranial EEG research. Progress in Neurobiology, 98(3), 279-301. https://doi.org/10.1016/j.pneuro bio.2012.06.008.

Lambon-Ralph, M.A., Jefferies, E., Patterson, K., Rogers, T.T. (2016). The neural and computational bases of semantic cognition. Nature Reviews Neuroscience, 18, 42-55. https://doi.org/10.1038 /nrn.2016.150.

Lichtheim, L. (1885). On aphasia. Brain, 7, 433-484. https://doi.org/ 10.1093/brain/7.4.433.

Liljestrom, M., Kujala, J., Stevenson, C., Salmelin, R. (2015). Dynamic reconfiguration of the language network preceding onset of speech in picture naming. Human Brain Mapping, 36(3), 1202-1216. https://doi.org/10.1002/hbm.22697.

Llorens, A., Trébuchon, A., Liégeois-Chauvel, C., Alario, F.X. (2011). Intra-cranial recordings of brain activity during language production. Frontiers in Psychology, 2. https://doi.org/10.3389/f psyg.2011.00375.

Llorens, A., Trébuchon, A., Riès, S., Liégeois-Chauvel, C., Alario, F.X. (2014). How familiarization and repetition modulate the picture naming network. Brain and Language, 133, 47-58. https://doi.org/10.1016/j.bandl.2014.03.010.

Llorens, A., Dubarry, A.S., Trèbuchon, A., Chauvel, P., Alario, F.X., Liègeois-Chauvel, C. (2016). Contextual modulation of hippocampal activity during picture naming. Brain and Language, 159, 92-101. https://doi.org/10.1016/j.bandl.2016.05.011.

Makris, N., \& Pandya, D.N. (2009). The extreme capsule in humans and rethinking of the language circuitry. Brain Structure and Function, 213(3), 343. https://doi.org/10.1007/s00429-008-0199-8.

Margulies, D.S., \& Petrides, M. (2013). Distinct parietal and temporal connectivity profiles of ventrolateral frontal areas involved in language production. Journal of Neuroscience, 33(42), 16,84616,852. https://doi.org/10.1523/jneurosci.2259-13.2013.

Maris, E. (2012). Statistical testing in electrophysiological studies. Psychophysiology, 49(4), 549-565. https://doi.org/10.1111/ j.1469-8986.2011.01320.x. 
Maris, E., \& Oostenveld, R. (2007). Nonparametric statistical testing of EEG and MEG data. Journal of Neuroscience Methods, 164(1), 177-190. https://doi.org/10.1016/j.jneumeth.2007.03.024.

Morlet, J. (1983). Sampling theory and wave propagation. In Chen, C. (Ed.) Issues in acoustic signal - image processing and recognition, NATO ASI series (series $f$ : computer and system sciences), (Vol. 1 pp. 233-261). Berlin: Springer.

Moscovitch, M., Cabeza, R., Winocur, G., Nadel, L. (2016). Episodic memory and beyond: the hippocampus and neocortex in transformation. Annual Review of Psychology, 67(1), 105-134. https://doi.org/10.1146/annurev-psych-113011-143733.

Papagno, C. (2011). Naming and the role of the uncinate fasciculus in language function. Current Neurology and Neuroscience Reports, 11(6), 553. https://doi.org/10.1007/s11910-011-0219-6.

Parlatini, V., Radua, J., Dell'Acqua, F., Leslie, A., Simmons, A., Murphy, D.G., Catani, M., de Schotten, M.T. (2017). Functional segregation and integration within fronto-parietal networks. NeuroImage, 146, 367-375. https://doi.org/10.1016 /j.neuroimage.2016.08.031.

Petrides, M. (2014). Neuroanatomy of language regions of the human brain. London: Academic Press.

Petrides, M., \& Pandya, D.N. (2009). Distinct parietal and temporal pathways to the homologues of Broca's area in the monkey. PLoS Biology, 7(8), e1000,170. https://doi.org/10.1371/journa 1.pbio. 1000170 .

Piai, V., Roelofs, A., Rommers, J., Maris, E. (2015). Beta oscillations reflect memory and motor aspects of spoken word production. Human Brain Mapping, 36(7), 2767-2780. https://doi.org/10.10 02/hbm.22806.

Piai, V., Anderson, K.L., Lin, J.J., Dewar, C., Parvizi, J., Dronkers, N.F., Knight, R.T. (2016). Direct brain recordings reveal hippocampal rhythm underpinnings of language processing. Proceedings of the National Academy of Sciences, 113(40), 11,366-11,371. https://doi.org/10.1073/pnas.1603312113.

Poeppel, D., Emmorey, K., Hickok, G., Pylkkänen, L. (2012). Towards a new neurobiology of language. Journal of Neuroscience, 32(41), 14,125-14,131. https://doi.org/10.1523/JNEUR OSCI.3244-12.2012.

Price, C.J. (2012). A review and synthesis of the first 20 years of PET and fMRI studies of heard speech, spoken language and reading. NeuroImage, 62(2), 816-847. https://doi.org/10.1016/j.neur oimage.2012.04.062.

Riès, S. K., Dhillon, R.K., Clarke, A., King-Stephens, D., Laxer, K.D., Weber, P.B., Kuperman, R.A., Auguste, K.I., Brunner, P., Schalk, G., Lin, J.J., Parvizi, J., Crone, N.E., Dronkers, N.F., Knight, R.T. (2017). Spatiotemporal dynamics of word retrieval in speech production revealed by cortical high-frequency band activity. Proceedings of the National Academy of Sciences, 114(23), E4530-E4538. https://doi.org/10.1073/pnas.1620669114.

Roelofs, A. (2014). A dorsal-pathway account of aphasic language production: the WEAVER++/ARC model. Cortex, 59, 33-48. https://doi.org/10.1016/j.cortex.2014.07.001.

Rogalsky, C., Poppa, T., Chen, K.H., Anderson, S.W., Damasio, H., Love, T., Hickok, G. (2015). Speech repetition as a window on the neurobiology of auditory-motor integration for speech: a voxelbased lesion symptom mapping study. Neuropsychologia, 71, 18-27. https://doi.org/10.1016/j.neuropsychologia.2015.03.012.

Rolston, J.D., \& Chang, E.F. (in press). Critical language areas show increased functional connectivity in human cortex. Cerebral Cortex, 1-8. https://doi.org/10.1093/cercor/bhx271.
Saur, D., Kreher, B.W., Schnell, S., Kummerer, D., Kellmeyer, P., Vry, M.S., Umarova, R., Musso, M., Glauche, V., Abel, S., Huber, W., Rijntjes, M., Hennig, J., Weiller, C. (2008). Ventral and dorsal pathways for language. Proceedings of the National Academy of Sciences, 105(46), 18,035-18,040. https://doi.org/10.1073/pn as.0805234105.

Schwartz, M.F., Faseyitan, O., Kim, J., Coslett, H.B. (2012). The dorsal stream contribution to phonological retrieval in object naming. Brain: A Journal of Neurology, 135(12), 3799-3814. https://doi.org/10.1093/brain/aws300.

Sheldon, S., \& Moscovitch, M. (2012). The nature and time-course of medial temporal lobe contributions to semantic retrieval: an fMRI study on verbal fluency. Hippocampus, 22(6), 14511466.

Sheldon, S., McAndrews, M.P., Pruessner, J., Moscovitch, M. (2016). Dissociating patterns of anterior and posterior hippocampal activity and connectivity during distinct forms of category fluency. Neuropsychologia, 90, 148-158. https://doi.org/10.1016/ j.neuropsychologia.2016.06.028.

Snodgrass, J.G., \& Vanderwart, M. (1980). A standardized set of 260 pictures: norms for name agreement, image agreement, familiarity, and visual complexity. Journal of Experimental Psychology: Human Learning and Memory, 6(2), 174. https://doi.org/10.1037 10278-7393.6.2.174.

Snodgrass, J.G., \& Yuditsky, T. (1996). Naming times for the Snodgrass and Vanderwart pictures. Behavior Research Methods, 28(4), 516-536. https://doi.org/10.3758/BF03200540.

Tremblay, P., \& Dick, A.S. (2016). Broca and Wernicke are dead, or moving past the classic model of language neurobiology. Brain and Language, 162, 60-71. https://doi.org/10.1016/j.bandl. 2016.08.004.

Uddin, L.Q., Supekar, K., Amin, H., Rykhlevskaia, E., Nguyen, D.A., Greicius, M.D., Menon, V. (2010). Dissociable connectivity within human angular gyrus and intraparietal sulcus: evidence from functional and structural connectivity. Cerebral Cortex, 20(11), 2636-2646. https://doi.org/10.1093/cercor/bhq011.

Ueno, T., Saito, S., Rogers, T.T., Lambon Ralph, M.A. (2011). Lichtheim 2: synthesizing aphasia and the neural basis of language in a neurocomputational model of the dual dorsal-ventral language pathways. Neuron, 72(2), 385-396. https://doi.org/10.1016/j.neur on.2011.09.013.

Wang, X.J. (2010). Neurophysiological and computational principles of cortical rhythms in cognition. Physiological Reviews, 90(3), 1195-1268. https://doi.org/10.1152/physrev.00035.2008.

Wang, H.E., Bénar, C. G., Quilichini, P.P., Friston, K.J., Jirsa, V.K., Bernard, C. (2014). A systematic framework for functional connectivity measures. Frontiers in Neuroscience, 8, 405.

Wernicke, C. (1969). The symptom complex of aphasia. In Cohen, R., \& Wartofsky, M. (Eds.) Proceedings of the Boston colloquium for the philosophy of science 1966/1968, (Vol. 4 pp. 34-97). Dordrecht: Springer. https://link.springer.com/chapter/10.1007/ 978-94-010-3378-7_2.

Wu, Y., Sun, D., Wang, Y., Wang, Y., Wang, Y. (2016). Tracing short connections of the temporo-parieto-occipital region in the human brain using diffusion spectrum imaging and fiber dissection. Brain Research, 1646, 152-159. https://doi.org/10.101 6/j.brainres.2016.05.046. 